\title{
A study of angular rate sensing by corona discharge ion wind
}

\author{
Van Thanh Dau ${ }^{1^{*}}$, Thien Xuan Dinh², Canh Dung Tran ${ }^{3}$, Tung Thanh Bui ${ }^{4}$ and Hoa Thanh Phan ${ }^{*}$ \\ ${ }^{1}$ Research Group (Environmental Health), Sumitomo Chemical. Ltd, Hyogo 665-8555, Japan \\ ${ }^{2}$ Graduate School of Science and Engineering, Ritsumeikan University, Shiga 525-8577, Japan \\ ${ }^{3}$ School of Mechanical and Electrical Engineering, University of Southern Queensland, Australia \\ ${ }^{4}$ University of Engineering and Technology, Vietnam National University, Hanoi, Vietnam \\ ${ }^{5} \mathrm{HaUI}$ Institute of Technology, Hanoi University of Industry, Hanoi, Vietnam \\ "Corresponding authors: dauv@sc.sumitomo-chem.co.jp, dauthanhvan@gmail.com; phanthanhhoa@haui.edu.vn
}

\section{ABSTRACT.}

We report an application of ion wind in angular rate sensing. A new design of a jet flow gyroscope using ion wind corona discharge is developed by conducting a numerical simulation and then an experimental analysis with regard to the advantages of a corona-discharge-based jet flow. Ion wind is generated by applying a high-voltage between a pin, as the discharge electrode, and a ring, as the reference electrode. The current-voltage characteristics of the discharge process by experimental works is set up as the boundary condition to describe the ion wind flow in the numerical simulation. A jet flow of ion wind is observed through an experiment and a simulation. When the gyroscope is subjected to an angular rate, the induced Coriolis force deflects the ion wind. This deflection is detected using four hotwires installed downstream of the working chamber behind the reference electrode. The experimental result show that the angular rate can be detected with a sensitivity of $4.7 \mu \mathrm{V} / \% / \mathrm{s}$. Because ion wind can be generated with minimum power and does not require any vibrating components, the device is robust, consumes low power, and is cost-effective.

Keywords: Ion wind; angular rate sensing; OpenFOAM; dielectric material 


\title{
A study of angular rate sensing by corona discharge ion wind
}

\author{
Van Thanh Dau ${ }^{1 *}$, Thien Xuan Dinh², Canh Dung Tran ${ }^{3}$, Tung Thanh Buil and Hoa Thanh Phan ${ }^{5}$ \\ ${ }^{1}$ Research Group (Environmental Health), Sumitomo Chemical. Ltd, Hyogo 665-8555, Japan \\ ${ }^{2}$ Graduate School of Science and Engineering, Ritsumeikan University, Shiga 525-8577, Japan \\ ${ }^{3}$ School of Mechanical and Electrical Engineering, University of Southern Queensland, Australia \\ ${ }^{4}$ University of Engineering and Technology, Vietnam National University, Hanoi, Vietnam \\ ${ }^{5} \mathrm{HaUI}$ Institute of Technology, Hanoi University of Industry, Hanoi, Vietnam \\ “Corresponding author: dauv@sc.sumitomo.co.jp, dauthanhvan@gmail.com; phanthanhhoa@haui.edu.vn
}

\section{ABSTRACT.}

We report an application of ion wind in angular rate sensing. A new design of a jet flow gyroscope using ion wind corona discharge is developed by conducting a numerical simulation and then an experimental analysis with regard to the advantages of a corona-discharge-based jet flow. Ion wind is generated by applying a high-voltage between a pin, as the discharge electrode, and a ring, as the reference electrode. The current-voltage characteristics of the discharge process by experimental works is set up as the boundary condition to describe the ion wind flow in the numerical simulation. A jet flow of ion wind is observed through an experiment and a simulation. When the gyroscope is subjected to an angular rate, the induced Coriolis force deflects the ion wind. This deflection is detected using four hotwires installed downstream of the working chamber behind the reference electrode. The experimental result show that the angular rate can be detected with a sensitivity of $4.7 \mu \mathrm{V} / \% / \mathrm{s}$. Because ion wind can be generated with minimum power and does not require any vibrating components, the device is robust, consumes low power, and is cost-effective.

\section{NOMENCLATURE}

$\begin{array}{llll}U & \text { Average ion wind velocity } & \varepsilon & \text { Permittivity of air } \\ I & \text { Corona discharge current } & q_{e} & \text { Elementary charge } \\ V & \text { Corona discharge voltage } & \omega & \text { Angular velocity } \\ \mu & \text { lon mobility } & L & \text { Length of hotwire } \\ q & \text { Charge density } & L & \text { Distance from ring electrode to hotwire } \\ E & \text { Electric field } & i_{h w} & \text { Heating current of hotwire } \\ f_{e} & \text { Electromagnetic force } & \lambda & \text { Thermal conductivity of air } \\ \rho & \text { Air Density } & \alpha & \text { Temperature coefficient of resistance of hotwire } \\ v & \text { Kinetic viscosity of air } & r_{t} & \text { Radius of turntable } \\ s & \text { Distance from pin electrode to ring electrode } & d & \text { Diameter of hotwire }\end{array}$

\section{Introduction}

A gyroscope is a type of inertial sensor that precisely measures the angular velocity and altitude angle. The main principle of gyroscopes is based on the effect of the Coriolis acceleration in the vibration of a reference proof mass [1]. In spite of high measurement performance as well as recent advancements in design and fabrication technology for these devices, gyroscopes with vibrating proof masses are fragile due to the oscillation of the mass element. Furthermore, the high cost for prototyping could affect the whole progress from a theoretical idea to the working samples. Meanwhile, fluidic-based gyroscopes do not require a proof mass, and therefore, do not suffer from any corresponding disadvantages. In such sensors, the reference movement comes from fluidic media, instead of a solidstate structure.

Several techniques are used to generate flow in fluidic-based gyroscope: (i) the natural-convection-based method from a locally heated region where the flow moves along the mass diffusion direction [2], or (ii) the thermalexpansion-based method induced by quickly heating gas [3]. While these approaches inherit advantages from microfabrication to reduce the size of the system, their drawbacks include low bandwidth and dependence on linear acceleration [4]; (iii) the third one is to create a synthetic jet using a piezoelectric diaphragm of a lead zirconate titanate (PZT) membrane whose vibration rectifies flow into a jet with an average velocity of several $\mathrm{m} / \mathrm{s}$ [5]-[9]; 
(iv) Instead of using PZT, jet flow is released from an electro-conjugate fluid using a high electric field created by a high voltage which is applied on electrodes submerged in the liquid. The electric field then generates electro hydrodynamic forces inside the pilot liquid [10], [11]. Whereas a gyroscope utilizing jet flows eliminates the effect of linear acceleration by force convection, its configuration is rather larger and complex [12]-[14].

Since the first publication on ion wind by Chattock [15] in 1899, many researchers have been attracted to apply the corona discharge phenomenon in various areas including propulsion technology [16], cooling applications [17], airflow control [18], and synthetic jet design [19]. With these applications, an electro-hydrodynamic (EHD) flow, also called ion wind, can be generated using high voltage corona discharges. The application of EHDs yields several distinguished advantages such as low weight, simplicity, robustness, lack of moving parts, and low power consumption. In this spirit, ionic wind corona discharge can be applied to develop angular rate sensors, where a flow is able to freely vibrate in three dimensional space under inertial forces.

Although the idea of using ion wind in this application has been mentioned previously, for example the patents [20], [21], not much technical documentation has been represented so far. Thus, in this work, a relevant design of an ion wind generating device and its application in angular rate sensing are presented. The numerical simulation of the present device is carried out for an initial design as well as a possible prediction of several other characteristics of the system. Analysis and investigated are performed in considering the properties of a gyroscope through an investigation of jet flow by experimental works.

Besides several above-mentioned advantages of the present approach based device such as low weight, simplicity, robustness, and low power consumption compared with other conventional gyros, instead of a research-grade tool, this work has designed and manufactured an ion wind gyroscope with inexpensive and commercially available components.

\section{Principle of the ion wind gyroscope}

In general, the main task in designing a fluidic angular sensor is to create a laminar jet flow that continuously floats inside a minimized space. In this work, the jet flow is generated using an electro-hydrodynamic force induced in a media of ionized air under a strong electric field between electrodes, including a pin and a ring (see Fig. 1). With the effect of the electric field, ions are displaced out of the vicinity of the pin toward the ring electrode. Along the displacement, ions transfer momentum to neutral molecules of air when colliding with them and drift the flow of air to a target.

In a point-ring configuration, the average velocity $(U)$ of ion wind is estimated, from the discharge current, by [22] as follows.

$$
U=\mathrm{k} \sqrt{I / \rho \mu},
$$

where $k$ is the coefficient and depends on the electrode discharge area and the inter-electrode distance, $\mu=1.6 \times 10^{-4}$ $\mathrm{m}^{2} \cdot \mathrm{V}^{-1} \cdot \mathrm{s}^{-1}$ the ion mobility, $\rho=1.2041 \mathrm{~kg} \cdot \mathrm{m}^{-3}$ the air density and $/$ the discharge current in $\mu \mathrm{A}$.

Once flow is generated in the device mounted on the rotating frame, it is affected by the Coriolis force. Indeed, the effect of the rotating frame on the flow is overwhelmed by the strong electric field that handles the flow along the distance from the pin electrode to the ring. After passing through the ring electrode in the working chamber, the flow is deflected by the Coriolis force as shown in Fig. 1. Inside the working chamber, this deflection is detected using four hotwires placed at a distance $L$ from the ring center. The deflection of flow can be determined as follows.

$$
\overrightarrow{d_{\omega}}=\iint \overrightarrow{a_{\omega}} d t=\iint 2 \vec{U} \times \vec{\omega} d t
$$

where $t$ is the time period while air flow shifts from the ring electrode to a plane perpendicular to the flow axis, where hotwires are set up; $U$ the average velocity calculated by $U=L / t$ and $\omega$ the angular velocity of the frame. As a result, the deflection of the air flow in the chamber on the direction normal to the flow axis is $d_{\omega}=\omega L^{2} / U$.

Letting $\beta$ be the gradient of the velocity profile of air flow on the plane of the hotwire, the deflection of air flow $\left(\Delta U_{\omega}\right.$ ) can be converted into a variation of air flow velocity by

$$
\Delta U_{\omega}=\beta \cdot d_{\omega}=\beta \omega \frac{L^{2}}{U}
$$




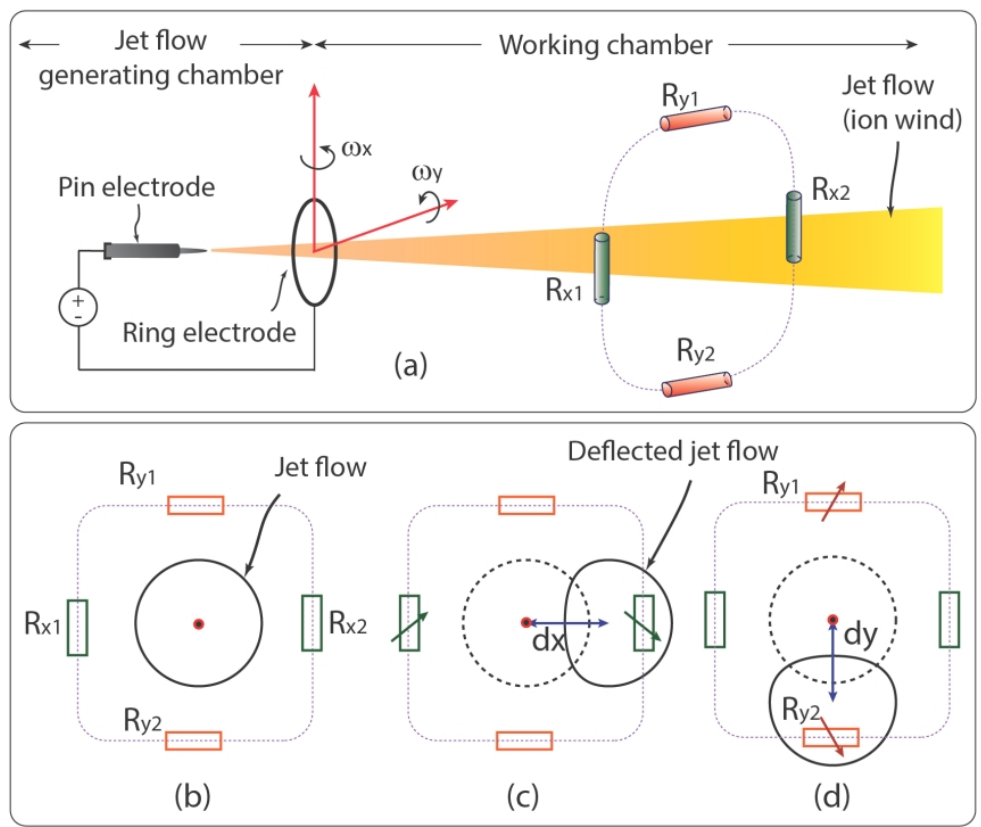

Figure 1. Principle of the ion wind gyroscope: (a) Jet flow generated by pin - ring electrodes reaches to the plane of hotwires; (b) Relative positions of jet flow when there is no applied angular rate, (c) there is applied angular rate around $x$ axis and $(d)$ around $y$ axis.

The flow deflection is then converted into the output voltage of hotwires.

For a hotwire heated by an electric current $i_{h w}$ and subjected to a variation of cooling air velocity $\Delta U_{\omega}$, the temperature variation of the hotwire is converted into a relative variation of resistance as follows [7]

$$
\Delta R=\frac{\lambda \pi l \alpha i_{h w}^{2} R_{H W}^{2}}{\left(\lambda \pi l N u-i_{h w}^{2} R_{H W} \alpha\right)^{2}} \cdot \frac{n N u}{U} \cdot \Delta U_{\omega}
$$

where $l$ is the typical length of the hotwire, $\alpha$ the temperature coefficient of the resistance, and $R_{H W}$ the reference resistance of the hotwire, $\lambda$ the thermal conductivity of the air; and Nu the Nusselt number representing the heat transfer coefficient between air flow and the hotwire.

Substituting Eq. (1) and (3) into (4) yields,

$$
\Delta \mathrm{R}_{\omega}=\frac{\lambda \pi l \alpha i_{h_{W}} \mathrm{R}_{H W 0}^{2}}{\left(\lambda \pi l N u-\mathrm{i}_{h w}^{2} \mathrm{R}_{\mathrm{HW} O} \alpha\right)^{2}} \cdot \frac{\rho \mu \mathrm{nNu}}{I \mathrm{k}^{2}} \cdot \beta \mathrm{L}^{2} \omega
$$

Equation (5) describes the relationship between the discharge current, applied angular velocity of the system, and the hotwire resistance. If a jet flow is self-similar and aimed at the center of the hotwire plane, a pair of hotwires $R_{X 1}$ and $R_{X 2}$ (or $R_{Y 1}$ and $R_{Y 2}$ ) is set up to form a Wheatstone half-bridge for measuring $\overrightarrow{\omega_{Y}}$ (or $\overrightarrow{\omega_{X}}$ ). Under the effect of $\overrightarrow{\omega_{Y}}$, resistors $R_{X 1}$ and $R_{X 2}$ are reversely changed, while $R_{Y 1}$ and $R_{Y 2}$ are identically affected, therefore, these bridges are independent from each other.

It is worth noting that the flow velocity can be represented by a discharge current (see Eq. (1)). Thus, once the dimension of the generated ion wind configuration is selected, the performance of the system can be simply predicted by the discharge current at the pin electrode and the heating current at hotwires.

\section{Numerical simulation}

A multi-physics numerical approach is used to analyze the flow characteristics of the considered system in which the Coriolis deflection is simulated using a rotating frame to generate the volumetric force in the momentum equation. The resulting model describes a multi physical problem including electrical field inducing the 
migration of ions within the inter-electrode region interacting with the dynamic flow of air in a chamber/channel.

\subsection{Governing equations and boundary conditions}

Charged ion winds generated by pin electrodes move under the effect of an electric field and drift air flow which, in turn, redistributes itself across the domain under consideration. Neglecting the conduction and diffusion of ions through the air, the charge density $q$ of ions, mainly caused by the ion $\operatorname{drift}(\mu \vec{E} q)$ and ion convection $(\vec{U} q)$ for the steady state, is governed by the equation of charge conservation and the Gauss' law, respectively as follows

$$
\begin{aligned}
& \nabla \cdot(\mu \vec{E} q+\vec{U} q)=0, \\
& \nabla \cdot(\nabla \emptyset)=-q / \varepsilon_{0},
\end{aligned}
$$

where $\mu$ is the mobility of positive and negative charges and, given by $\mu=1.6 \times 10^{-4} \mathrm{~m}^{2} \cdot \mathrm{V}^{-1} \cdot \mathrm{s}^{-1} ; \vec{U}$ the velocity of air drifted by the motion of charge and $\epsilon_{0}=8.854 \times 10^{-12} \mathrm{C} \cdot \mathrm{V}^{-1} \cdot \mathrm{m}^{-1}$ the permittivity of the air. The electric field $\vec{E}$ is a function of the electric potential and is defined by $\vec{E}=\nabla V$.

The corona discharge is set up as a boundary condition on the electrodes assuming that the surface charge density $\mathrm{q}_{\mathrm{s}}$ is determined as a function of the discharge current $I$ which is measured from the I-V experimental curve as follows.

$$
\mathrm{q}_{\mathrm{s}}=I /\left(\mu E_{\mathrm{on}} A\right)
$$

where $A$ is the total area of the electrodes creating an electric field with a magnitude greater than the onset $E_{\mathrm{on}}=3.23 \times 10^{6} \mathrm{~V} / \mathrm{m}[22]$. Meanwhile, the potentials on the pin and ring electrodes are equal to the applied voltages $V$ and 0 , respectively. The Neumann condition is applied at the edges of the model to close the simulation domain.

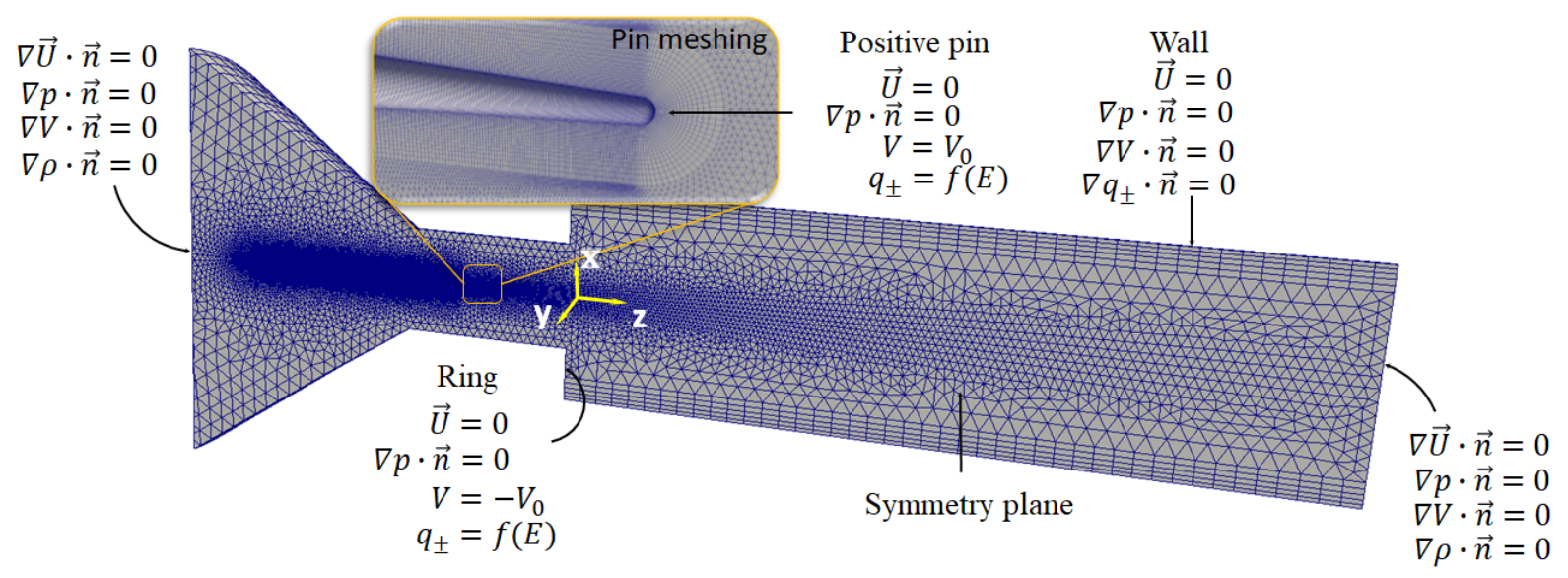

Figure 2. Simulation model with meshing and boundary conditions.

For the gas motion, in order to describe the self-generating flow system, no particular flow field condition was applied except the nonslip condition on the electrodes and the wall. The dynamics of a steady state incompressible air flow are governed by the Navier-Stokes and the continuity equations as follow

$$
\begin{aligned}
& \nabla \cdot(\overrightarrow{U U})-\nabla \cdot(\nu \nabla \vec{U})=-\nabla p+\frac{f_{e}}{\rho}-2 \rho \vec{\omega} \times \vec{U} \\
& \nabla \cdot \vec{U}=0
\end{aligned}
$$

where the term $2 \rho \vec{\omega} \times \vec{U}$ in Eq. (9) represents the Coriolis's apparent acceleration in a rotating frame with angular velocity $\vec{\omega} ; p$ pressure; $v=15.7 \times 10^{-3} \mathrm{~m}^{2} \cdot \mathrm{s}^{-1}$ kinematic viscosity and $\rho=1.2041 \mathrm{~kg} \cdot \mathrm{m}^{-3}$ air density. $f_{e}$ is the electromagnetic force acting on the plasma and consists of the Coulomb, permittivity gradient, dielectrophoretic and electrocostriction forces. In this work, since only air with a limited temperature 
gradient is considered, the last three forces are neglected. The only electromagnetic force considered is the Coulomb one and it is given by

$$
f_{e}=q \vec{E}
$$

using corona discharge as the boundary condition of charge density on the tips of the electrodes given by the measured current-voltage (I-V) characteristics of the corona as presented in section 4.2.1. Details of the boundary conditions are presented in Fig. 2.

In the simulation model, the spherical radius of the pin tip is $80 \mu \mathrm{m}$ and the distance from the pin to the ring is $7 \mathrm{~mm}$. The domain under consideration includes a cylinder chamber of jet flow with a diameter of 5 $\mathrm{mm}$ and length of $10 \mathrm{~mm}$ and a working chamber having a diameter of $12 \mathrm{~mm}$. The model consisting of 185 thousands mesh elements is described in Fig. 2.

The solutions of Eqs. (7)-(12) together with the boundary conditions are obtained using our solver, which was developed based on the finite volume library OpenFOAM, and the results are discussed in the next section.

\subsection{Numerical results and discussion}

Figure 3a shows that the generated jet flow is maintained downstream and away from the pin electrode. A jet flow of ions is generated by a strong electric field at the tip of electrode pin, and the ring electrode defines the movement of charged particles along the electric field line. A visualization of the ion wind by smoke particles introduced in the device from the pin side is also presented in Fig. $3 \mathrm{~b}$ whose video clip is attached as a supplementary document.

The velocity profile $U_{z}$ with respect to the $x$ direction (named as $U_{z / x}$ ) at several positions of distance $L$ from the nozzle is presented in Fig. 4. Results show that the profile $U_{z / x}$ is axially symmetrical inside the working chamber. The velocity in the profile reduces with the increase of distance $L$ but the jet form is maintained. This velocity profile is used as a preliminary reference to install hotwires in our experimental device, which is presented in section 4 . As shown in Fig. 4, hotwires are set up at a distance of $2.5 \mathrm{~mm}$ from the center of the flow axis

to maximize the deflection of the flow when the device is mounted on a rotating turntable. Results also show that a hotwire installed further from the central axis of the jet flow chamber detects larger deflections of flow but with less sensitivity and vice versa.

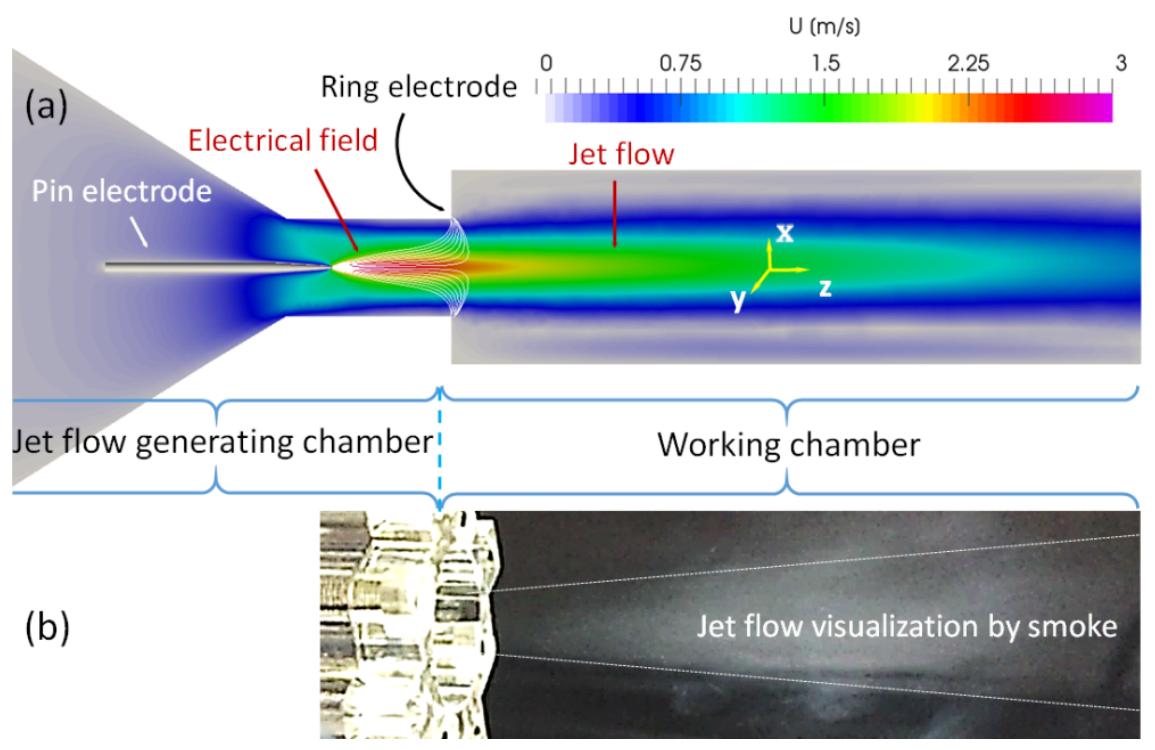

Figure 3. Simulation results: (a) lon wind generated by the point-ring configuration and (b) Jet flow visualized by smoke (see supplementary document - attached video clip). The discharge conditions are set up by $\mathrm{V}=$ $6.2 \mathrm{kV}, \mathrm{I}=2 \mu \mathrm{A}$ and electrode distance $=7 \mathrm{~mm}$. 


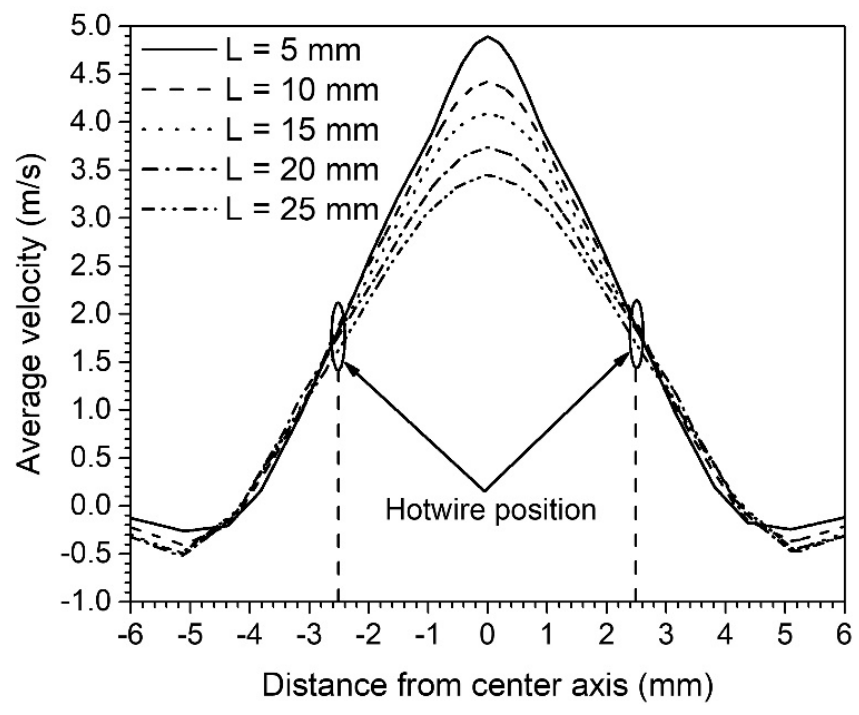

Figure 4. Simulation results: velocity profile $U_{z / x}$ with respect to $x$ direction plotted versus the distance from the center axis of the jet flow chamber at 5 positions from the ring electrode ( $L=5,10,15,20$ and $25 \mathrm{~mm}$ ). The discharge conditions are set-up by $\mathrm{V}=6.2 \mathrm{kV}, \mathrm{I}=2 \mu \mathrm{A}$ and electrode distance $=7 \mathrm{~mm}$.

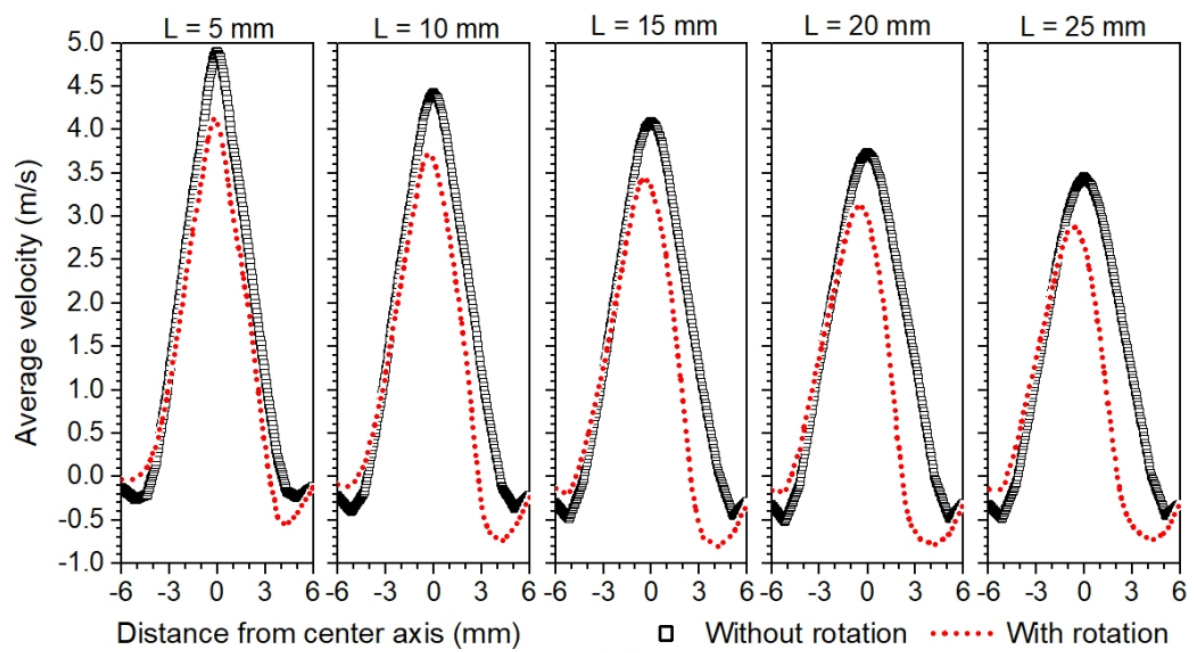

(a)

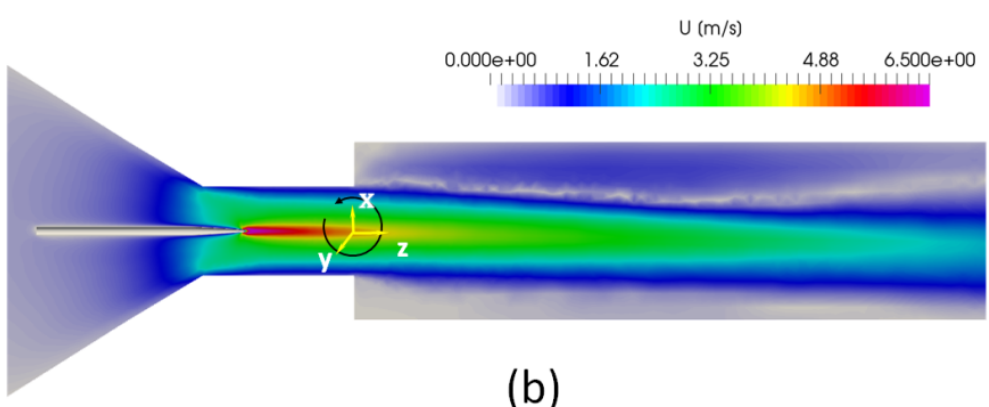

Figure 5. Simulation results: (a) Velocity profile $U_{z / x}$ of jet flow on the cross-section of the jet flow chamber at five positions ( $L=5,10,15,20$ and $25 \mathrm{~mm}$ ) along $z$ axis when the device is at rest (black lines) and when rotating with an angular velocity $\omega_{y}=5 \mathrm{rpm}$ (red lines); and (b) velocity contour of the device with an angular velocity $\omega_{y}=5 \mathrm{rpm}$. The discharge current $\mathrm{I}=2 \mu \mathrm{A}$. 
Indeed, the simulation is repeated while the device rotates with an angular velocity of $\omega_{Y}=5 \mathrm{rpm}$ around $\mathrm{Y}$ axis as described in Figs. 3 and 5b. The velocity contour shown in Fig. $5 \mathrm{~b}$ depicts that the flow deflects downward. Furthermore, the velocity profiles $U_{z / x}$ of jet flow at five positions $L=5,10,15,20$ and $25 \mathrm{~mm}$ from the ring electrode in Fig. 5a show that the deflection of jet flow gradually increases at the further positions from the nozzle. This numerical observation is confirmed by Eq. (3). Therefore, with rotation about $y$ axis, the flow deflects downward and the velocities of jet flow at the two hotwires $R_{x 1}$ and $R_{x 2}$ (see Fig. 1) change oppositely as shown in Fig. $5 \mathrm{a}$.

Similarly, the velocity profile $U_{z}$ with respect to the $y$ direction (named as $U_{z / y}$ ) along the $z$ axis is also considered and the numerical results are provided in Figs. 6a, b. While the velocity contour of flow on the cross-section is flat on the upper half of the cross-section (Fig. 6a), the velocity profile $U_{z / y}$ is symmetric about the $z$ axis as shown in Fig. 6b. Therefore, the flow velocities $U_{z / y}$ at the hotwires $R_{y 1}$ and $R_{y 2}$ change similarly, which behaves differently from the change of $U_{z / x}$ at the hotwires $R_{x 1}$ and $R_{x 2}$ as aforementioned.

Furthermore, since the velocity variation of the flow at the hotwires $R_{y 1}$ and $R_{y 2}$ is the same, the effect of $\omega_{Y}$ on $Y$ hotwires can be eliminated by introducing a conventional electric circuit that can form a half Wheatstone bridge using a pair of $Y$ hotwire. Such circuit is commonly used in sensing techniques as reported in literature.

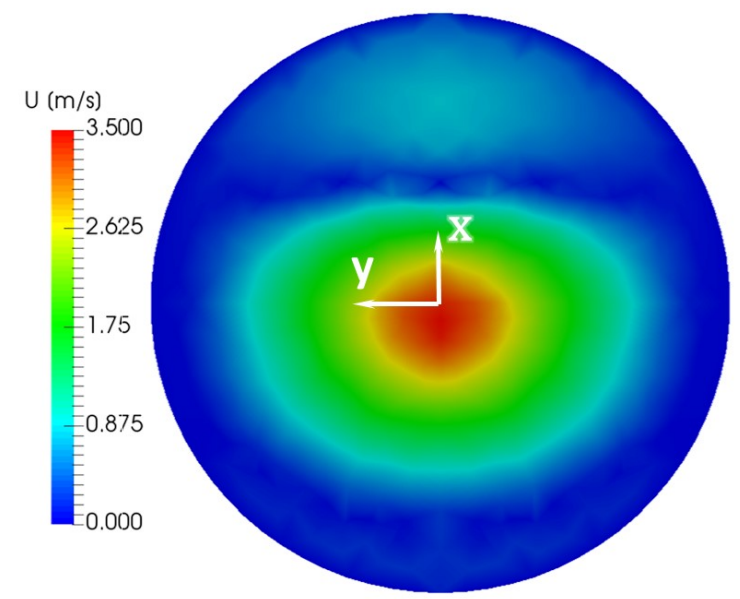

(a)

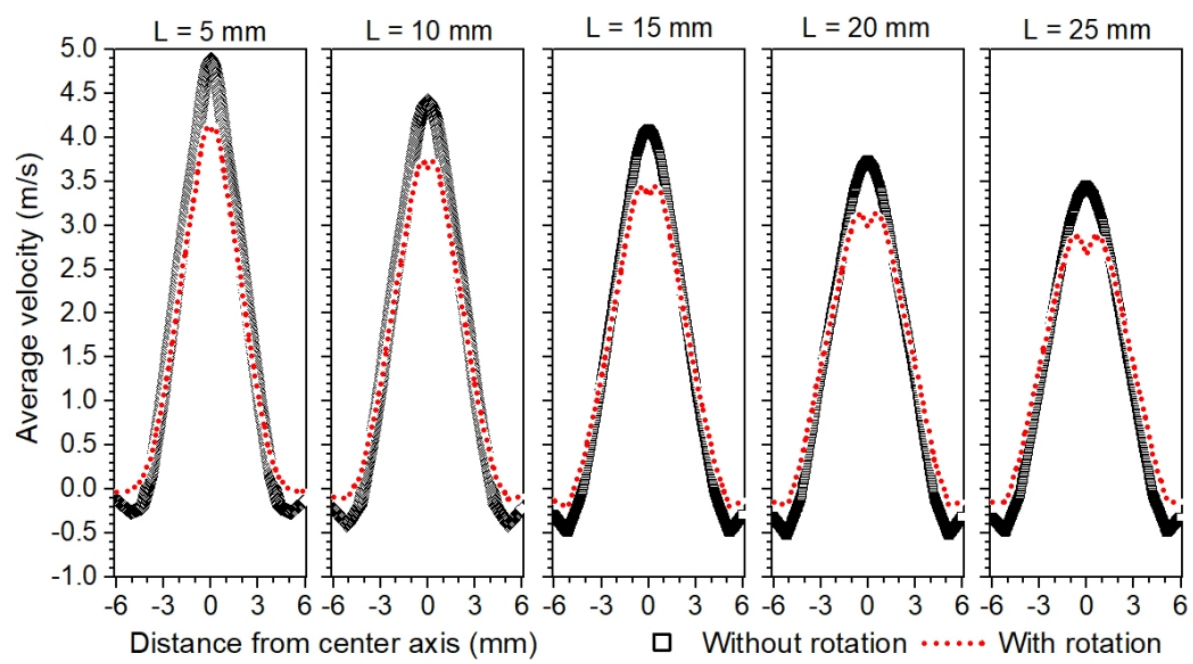

(b)

Figure 6. Simulation results: (a) Velocity contour of flow on the cross-section ( $x-y$ plane) with angular velocity $\omega=5 \mathrm{rpm}$-and (b) Velocity profile $U_{z / y}$ of jet flow on the cross-section of the jet flow chamber at five positions ( $L=5,10,15,20$ and $25 \mathrm{~mm}$ ) when the device is at rest (black lines) and when rotating with an angular velocity $\omega=5 \mathrm{rpm}$ (red lines). The discharge current $\mathrm{I}=2 \mu \mathrm{A}$. 


\section{Experiment}

\subsection{Design and experimental setup}

The designed configuration consists of two chambers of cylindrical shape connected to each other: an ion chamber where ion wind is generated and a working/sensing chamber (see Figs. 7a and 8a). The dimensions ( $\phi \times h$ with $\phi$ : diameter and $h$ : length) of the ion and working chambers are $5 \mathrm{~mm} \times 10 \mathrm{~mm}$ and $12 \mathrm{~mm} \times 15 \mathrm{~mm}$, respectively, which are similar to those of the simulation model.
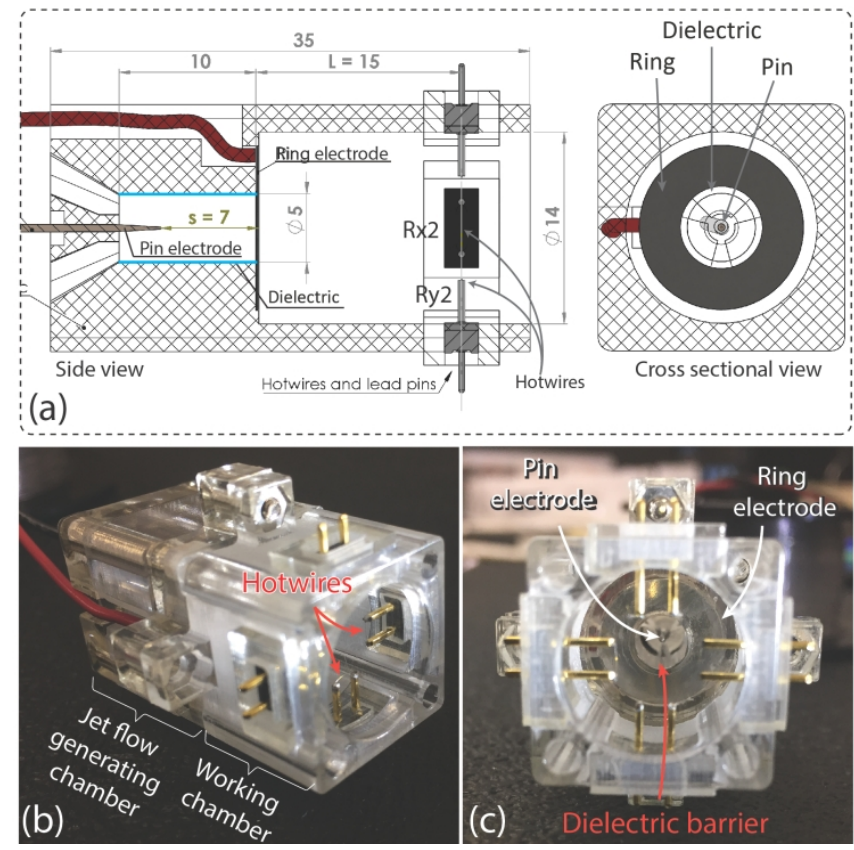

Figure 7. Experimental work: (a) Schema of the proposed sensor and (b, c) A prototype of sensor with 4 hotwires.
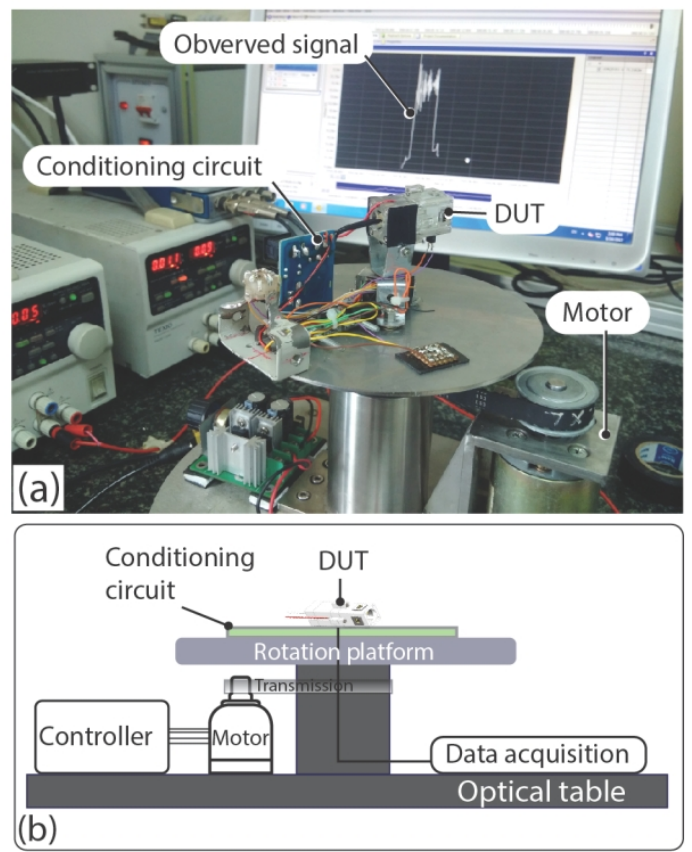

Figure 8. Experimental work: (a) Schema of the proposed sensor mounted on a turntable; and (b) Block diagram of the measurement set up. 
A pin of stainless steel SUS304 with a diameter of $0.4 \mathrm{~mm}$ and $8 \mathrm{~mm}$ long is set up with an optimized distance from the ring. A spherical pin tip with a radius of $80 \mu \mathrm{m}$ and a pin whose length is chosen for the sake of easy installation is used. The ring, made also of SUS304, has dimensions (inner diameter $\times$ outer diameter $\times$ thickness) of $6 \mathrm{~mm} \times 10$ $\mathrm{mm} \times 0.1 \mathrm{~mm}$. The electrodes are assembled into a dielectric frame, and the ring is hidden from the line-of-sight of the pin in order to prevent electric sparks as well as to further focus on the generated ion wind as shown in Fig. 7a and Fig. 8b. The position of the ring electrode with reference to the dielectric barrier affects the ion wind generation. For example, if the ring electrode is located far apart the barrier, a very high voltage will be necessary to generate the ion wind. In the worst case in which the dielectric barrier may eventually screen out the electric field, the direct discharge will not be possible. On the contrary, if the dielectric barrier is close to the plane of the ring electrode, or not intruding the line-of-sight between electrodes, its effects on the ion wind generation and spark prevention diminish. In our design, the ring is located at a distance of $0.5 \mathrm{~mm}$ behind the dielectric wall (Fig. 7a and Fig. 8b). A high-voltage generator, with capability of generating up to $10 \mathrm{kV}$, is connected to the pin and ring electrodes. A closed-loop control system is set-up to maintain a desired discharge current sensed at the negative electrode by using an adjustable current unit. The insulation between electrodes is guaranteed and evidenced by an insignificant leak current (less than $10 \mathrm{nA}$ ) which is observed between the electrode contact points.

In the working chamber, four tungsten hotwires (W-461077, Nillaco Ltd) with diameter of $20 \mu \mathrm{m}$ and length of 2.54 $\mathrm{mm}$ each are installed at a distance of $15 \mathrm{~mm}$ downstream from the ring using lead pins (Preci-Dip) like the hotwire holders (see Figs. $7 \mathrm{a}$ and $8 \mathrm{a}, \mathrm{b}$ ). In their plane, the hotwires are equally positioned $2.5 \mathrm{~mm}$ from the flow axis, i.e., the distance between two opposite hotwires is $5.0 \mathrm{~mm}$ as being optimized from simulation work in Fig. 4 .

The hotwires, whose resistance and temperature coefficient of resistance (TCR) are $0.453 \Omega$ and $4500 \mathrm{ppm} /{ }^{\circ} \mathrm{C}$, respectively, are connected to an electric circuit which is integrated into the device to capture signals (Fig. 7b, c). All acquired data are recorded and monitored by a LabVIEW DAQ6220 data acquisition system while the average velocity of air flow is determined from the hotwire voltage using a self-developed C-code routine (Fig. 8c, d). Additionally, a thermal anemometer (ISA-70, Sibata) is also set up at a relevant position, $50 \mathrm{~mm}$ away from the ring electrode, normal to the outlet of the device to measure the flow parameters. The measurement is recorded at 1 $\mathrm{Hz}$ in the room condition of temperature and pressure and the obtained results are recorded every 60 seconds.

The effect of the Coriolis force on the device is analyzed using a self-developed turntable, which comprises an aluminum disc with diameter of $10 \mathrm{~cm}$. The turntable is driven by a direct current motor and the angular velocity is monitored by an integrated encoder (Tsukasa Electric Ltd). A microcontroller PIC18F4520 (Microchip Ltd.) is used to generate output signals via pulse width modulation (PWM) while a H-bridge circuit of IC L298N is used as a motor driver and is controlled by two output voltages from PWMs of the PIC18F4520 for the direction and velocity of the motion. The device is mounted at the center and connected to the outer circuit through a slip-ring mechanism installed along the center of the turntable, which allows the electrical system to work safely while the turntable rotates. The turntable is tested with maximum revolutions of $10 \mathrm{rpm}$. Experimental results will be discussed in the next sections.

\subsection{Experimental results}

\subsubsection{I-V characteristics of corona discharge of the device}

The current - voltage $(I-V)$ characteristic of the corona discharge is examined by gradually increasing voltage and recording current. As the voltage increases, charged species (electrons and ions) are accumulated at the pin tip. When the voltage $V$ overcomes the inceptive voltage $V_{0}$, the current appears, indicating that numerous ions gain enough momentum to overpass the inter-electrode space, and the ion wind starts flowing inside the device. Since the ignition of the corona discharge is a highly complex phenomenon, the I-V characteristic measured by this experiment is used to set up the boundary condition of charge density on the tips of electrodes as presented in Eq. 9. The onset voltages in this experiment, with positive pin electrode and ground ring electrode, are $3.28 \mathrm{kV}$ and 5.33 $\mathrm{kV}$ for inter-electrode distances of $4 \mathrm{~mm}$ and $7 \mathrm{~mm}$, respectively.

Depending on the corona discharge configuration, its $I-V$ characteristic can be expressed by either Townsend relationship $(I / V \propto V)$ or $\sqrt{I} \propto V$. The empirical Townsend relation was originally found in coaxially cylindrical configurations and has been efficiently proven for point-to-ring configuration. On the other hand, the $\sqrt{I} \propto V$ relation fits better with configurations containing a bipolar discharge or positive corona in a limited space [23]. In this work, with the presence of a small dielectric barrier at the ring electrode, the designed configuration is slightly 
different from prior ones and then may not fit with any of the two relationships aforementioned. Hence, our experimental data is approximated using the general relation $\mathrm{I}^{1 / \mathrm{n}} \propto \mathrm{V}$, in logarithm form it is shown as follows.

$$
\log (\mathrm{I})=\mathrm{n} \times \log \left(\mathrm{V}-\mathrm{V}_{0}\right)+\log \mathrm{C}_{0}
$$

where $I$ is the discharge current and $C$ a constant depending on the electrode geometry and the ion mobility $\mu$. The inceptive voltage is usually selected with a current less than $1 \mu \mathrm{A}[24]$.

The I-V characteristics of ion wind measured by experiment with two inter-electrode gaps of $7 \mathrm{~mm}$ and $4 \mathrm{~mm}$ plotted in the logarithm form of $(I)$ and $\left(V-V_{o}\right)$ in Fig. 9a show a linear relationship with the slopes $(n)$ of 0.47 and 0.56 , respectively.

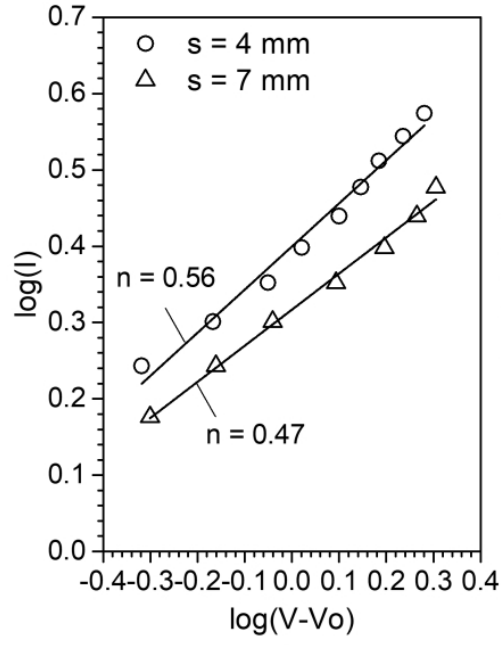

(a)

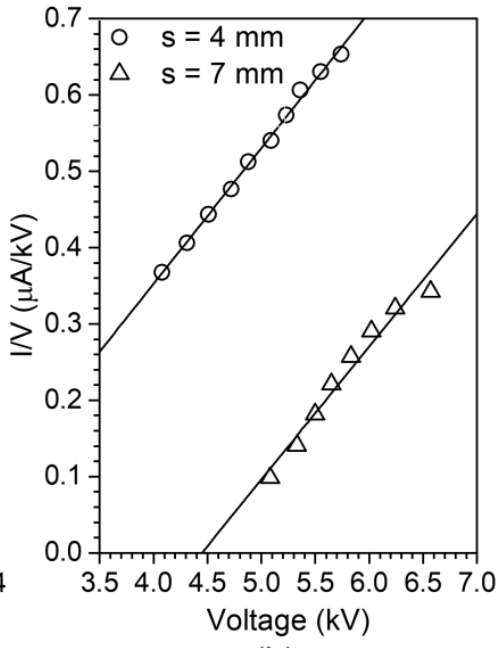

(b)

Figure 9. I-V characteristics using two inter-electrode distances of $4 \mathrm{~mm}$ and $7 \mathrm{~mm}$ with positive pin electrode and ground ring electrode: The experimental $I-V$ characteristics is expressed by the general relation $\left(I^{1 / n}\right.$ $\propto$ ) (a) and by Townsend relation (b).

For the comparison, these experimental data expressed by the Townsend relation $(I / V \sim V)$ are also shown in Fig. 6b. It can be observed that the general relation $I^{1 / n} \propto V$ better describes the $I-V$ characteristic of the present configuration with a coefficient of determination (R-square) for linear fitting higher than 0.986. Meanwhile the linear fitting using Townsend relation reached a R-square of 0.965 .

\subsubsection{Measuring velocity of the ion wind by anemometer.}

The velocity of ion wind $(U)$ measured by the conventional anemometer $50 \mathrm{~mm}$ downstream from the ring electrode with respect to the discharge current for two inter-electrode distances of $4 \mathrm{~mm}$ and $7 \mathrm{~mm}$ shows a linear relation in the form $(U \propto \sqrt{I})$ in Fig. 10. This result is in good agreement with numerical simulation, which are repeated using the I-V relation in Fig. 9 as the boundary condition. The results depict that the linear relation is steeper with the increase of inter-electrode distance. This can be explained as follows. With a larger inter-electrode gap, a higher voltage is required to maintain the discharge current as usual. Hence, ions are accelerated through a longer path from the pin to the ring electrodes.

This linear relation between velocity and the square root of discharge current $(U \propto \sqrt{I})$ can be expressed by Eq. (1). In order to establish Eq. (1), the coefficient $k$ is determined by fitting the data in Fig. 10 using $\mu=1.6 \times 10^{-4}$ $\mathrm{m}^{2} \cdot \mathrm{V}^{-1} \cdot \mathrm{s}^{-1}, \rho=1.2041 \mathrm{~kg} \cdot \mathrm{m}^{-3}$, and $I$ in $\mu \mathrm{A}$. The obtained values of $k$ are $0.023 \mathrm{~m}^{-1 / 2}$ and $0.014 \mathrm{~m}^{-1 / 2}$ for inter-electrode distances of $7 \mathrm{~mm}$ and $4 \mathrm{~mm}$, respectively. Thus, with an established Eq. (1), the ion wind velocity can be determined by the measured corona discharge current or voltage.

It is worth noting that the values of $k$ used in this research are higher than those in several published works, for example the value of $k$ was around $0.003 \mathrm{~m}^{-1 / 2}$ for an point-to-cylinder configuration and ion wind measured at similar downstream distance in [25], which means that a higher discharge current was required to generate the 
same velocity of ion wind. In this case, the presence of a dielectric barrier around the ring may contribute to reduce the discharge current in our case. We will address this interesting feature in our future work.

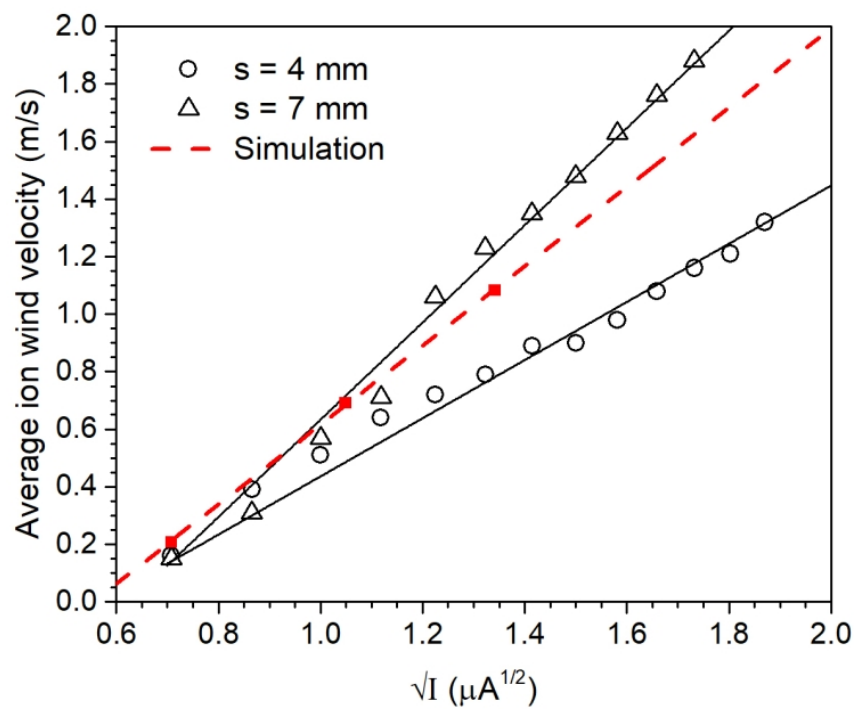

Figure 10. Experimental results: Velocity of ion wind plotted versus the discharge current for two interelectrode distances of $4 \mathrm{~mm}$ and $7 \mathrm{~mm}$. Simulation result is with electrode distance $\mathrm{s}=7 \mathrm{~mm}$.

\subsubsection{Performance of hotwires}

After ion wind is generated in working chamber as observed by the anemometer, the velocity of ion wind is investigated using hotwires as sensors. As described in section 4.1, the velocity of the ion wind by the hotwires is determined locally at defined positions where hotwires are set up on a circle with radius of $2.5 \mathrm{~mm}$ from the flow axis as shown in section 3.2 and Fig. 4.

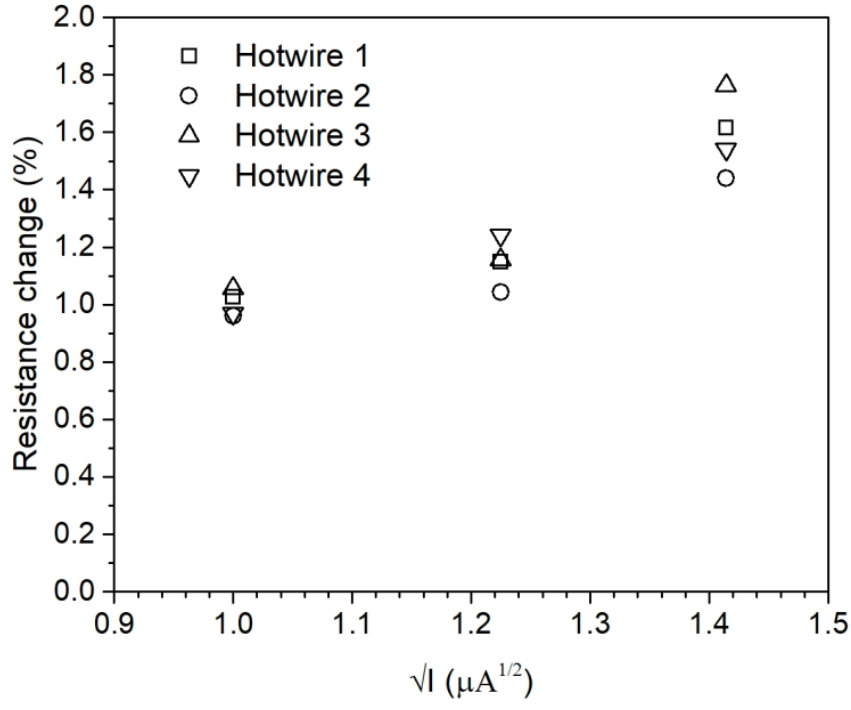

Figure 11. Experimental resistance values of four hotwires plotted versus the discharge current.

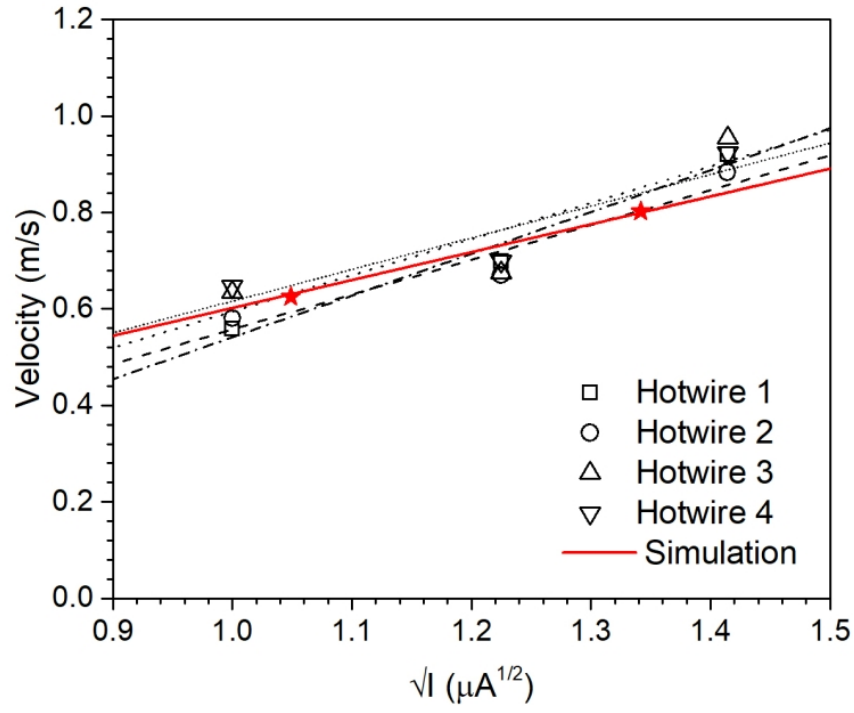

Figure 12. Experimental velocity values of ion wind converted from the resistance variation of four hotwires plotted versus the discharge current. Simulation parameters are similar to those in Fig. 10.

In this work, four hotwires are heated by a constant current $i_{h w}$ of $100 \mathrm{~mA}$. During the experiment, the temperatures of the hotwires change with the discharge current generating ion wind flow. The resistance variations of the hotwires are shown in Fig. 11, where the resistance of the hotwires increases with higher discharge current. 
The resistance variations of the hotwires is then converted to the ion wind velocity by comparing the heat transfer coefficient of forced convection, $0.24+0.56 R e^{0.45 \lambda} / D$, from the generated ion winds with that of natural convection $1.02 R a^{0.1} \lambda / D$ from a still air [26], where $\lambda$ is the thermal conductivity, Ra the Rayleigh number, $d$ the effective diameter of the hotwire and $R e=U D \rho / \mu$ the Reynolds number. The process is carried out using an subroutine C-code which was detailed in our recent publications [27]-[29].

The velocity of ion wind obtained by the conversion plotted with respect to the square root time of the discharge current $\sqrt{I}$ in Fig. 12 depicts that the ion wind velocity linearly increases with increasing $\sqrt{I}$. This observation is in very good agreement with the results by the conventional anemometer in Fig. 10. Moreover, experimental results in Fig. 12, which are consistent with numerical result, also show the ion wind velocity at four hotwire positions is nearly the same as observed by the simulation in section 3.2 and Fig. 4 because (i) the jet flow of the ion wind is axially symmetric and (ii) the hotwires are installed symmetrically on a plane perpendicular to the jet flow as shown in Fig. 1.

Finally, it is worth noting that, whereas the discharge current by ion wind is only several $\mu$ As, the applied current to heat the hotwires are much higher (order of $10^{5}$ in magnitude). Thus, the effect of the charged species (electrons and ions) from discharge current on the hotwire voltages is insignificant and therefore, the voltage calculated on the hotwires is based on the cooling effect of the ion wind flow.

\subsubsection{Application in sensing angular rate}

With a jet flow, the device can be used as a microfluidic gyroscope to detect angular rate. The device is investigated when it is horizontally mounted on a turntable locating the ring electrode as close as possible to the center of the turntable.

The experiment works with a corona discharge voltage of $5 \mathrm{kV}$ and $\mathrm{X}$ hotwires heated by a current $i_{\text {hw }}$ of $150 \mathrm{~mA}$. The output voltage is offset to initial values when the turntable is at rest. Fig. 13 depicts the output voltages on hotwires $X_{1}$ (Top figure) and $X_{2}$ (Bottom figure) recorded with respect to time when the turntable rotates at $5 \mathrm{rpm}$. Results show that the output voltages on hotwire $X_{1}$ and $X_{2}$ are in opposite value and vibrate over a range of values from $+116 \mu \mathrm{V}$ (standard deviation, $\pm 5 \%$ ) and $-142( \pm 5 \%) \mu \mathrm{V}$.
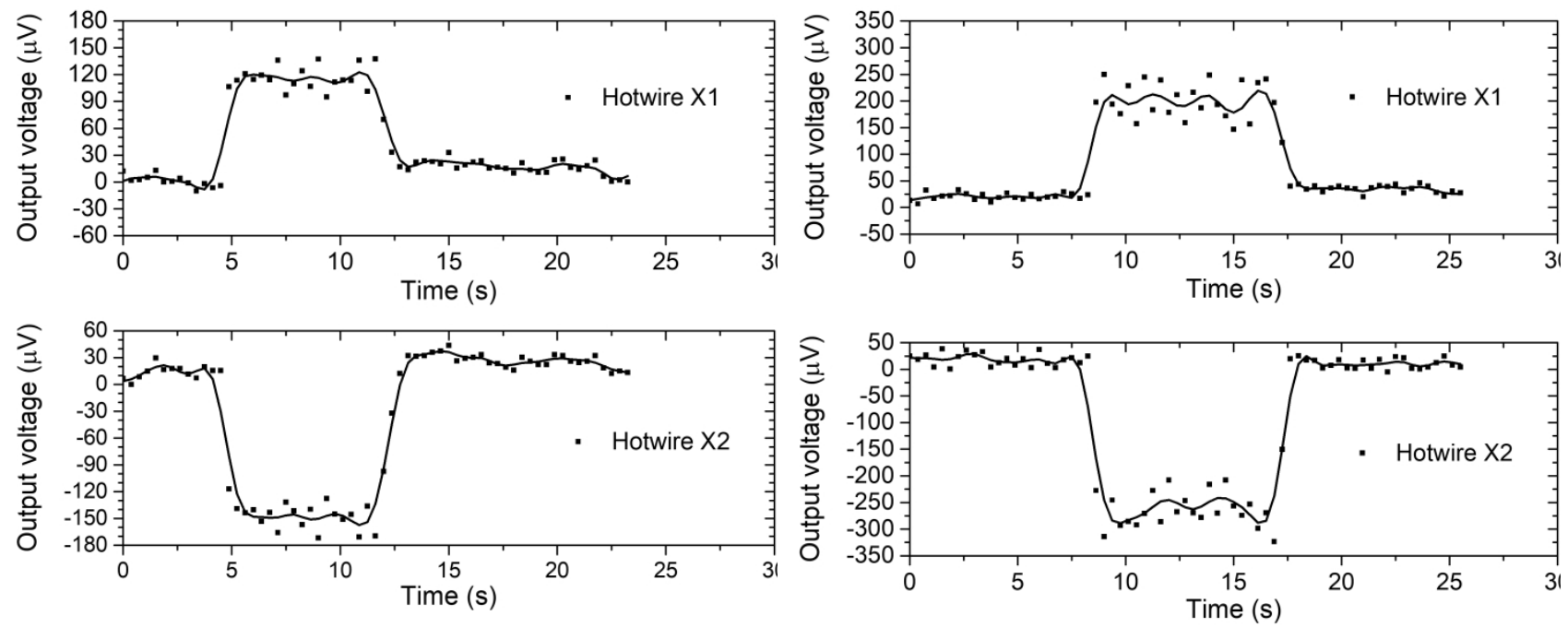

Figure 13. The time dependent output voltage measured at hotwire $X_{1}$ (top figure) and hotwire $X_{2}$ (bottom figure) by the experiment with a rotation of turntable of $5 \mathrm{rpm}$.
Figure 14. The time dependent output voltage measured at hotwire $X_{1}$ (top figure) and hotwire $X_{2}$ (bottom figure) by the experiment with a rotation of turntable of $10 \mathrm{rpm}$. 


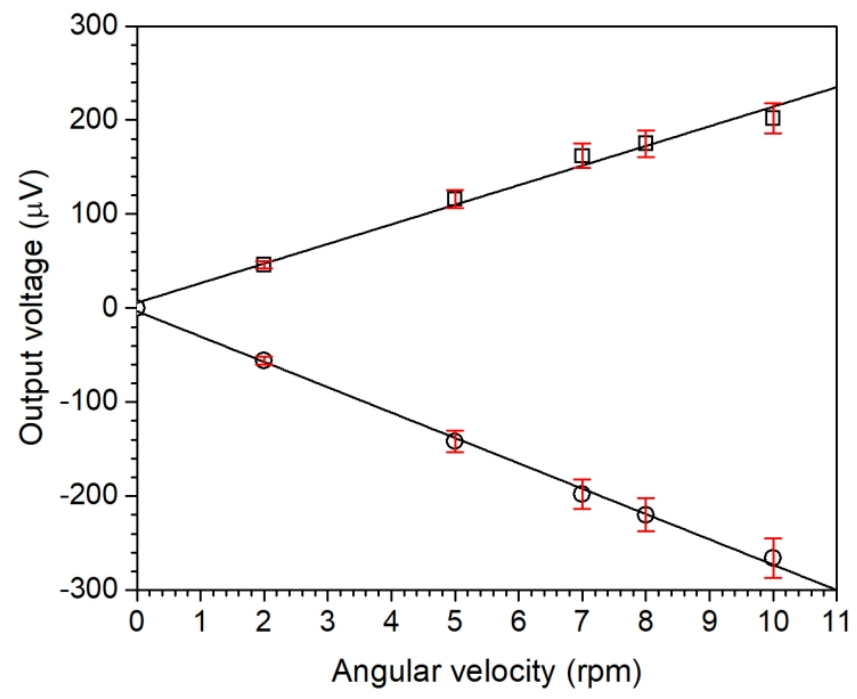

Figure 15. Output voltage on hotwires $X_{1}$ and $X_{2}$ at various rotating speed. The error bar is plotted with $\pm 8 \%$ standard variance.

The experiment is repeated with the angular velocity of the turntable at $10 \mathrm{rpm}$. The curves of output voltage measured on hotwires $X_{1}$ and $X_{2}$, are similar to ones of $5 \mathrm{rpm}$ but on the range of values from 202 ( $\pm 8 \% \mu \mathrm{V}$ to $266( \pm 6 \%) \mu \mathrm{V}$ as presented in Fig. 14. Results also show that the output voltage is almost double compared with the one in Fig. 13. This can be explained by the symmetry of the ion wind and the linear relationship of the velocity of ion wind flow versus the distance from the flow center to the position of hotwires as presented in Fig. 4.

Generally, the relation between output voltages on hotwires $X_{1}$ and $X_{2}$ are plotted in Fig. 15 where the turntable rotates from $2 \mathrm{rpm}$ to $10 \mathrm{rpm}$. The output voltages change almost linearly with the applied voltage, and the sensitivity of the hotwires $X_{1}$ and $X_{2}$ are calculated as $3.8 \mu \mathrm{V} / \% / \mathrm{s}$ and $4.7 \mu \mathrm{V} / \% / \mathrm{s}$, respectively.

Several notable issues found through our experiments help evaluate and improve the present device, as well as yield our future works, including

- The experiment mentioned in section 4 with inter-electrode distance of $7 \mathrm{~mm}$ is repeated but using a different configuration with grounded pin and positive ring electrode, thus negative ion wind instead of positive one is generated with a velocity of $1.3 \mathrm{~m} / \mathrm{s}$ observed by the anemometer at its position ( $50 \mathrm{~mm}$ away from the ring electrode). With this configuration, experimental results in Fig. 16 show that a linear relationship between the velocity of flow and the square root of the discharge current (U $\propto \sqrt{I}$ ) fitting by Eq. (1) with the coefficient $k$ of $0.007 \mathrm{~m}^{-1 / 2}$, is smaller than that of positive corona in section 4.2.2. However, experiment confirms that it is more comfortable to handle a negative corona discharge because it is stable on a larger range of discharge voltages than the positive corona. In addition, although the positive corona generates stronger ion winds, it would produce unexpected ozone and, therefore, contamination from the electrochemical process due to the humid air surrounding the electrodes [30], [31]. Thus, a relevant selection of the corona (positive or negative) should be based on the resources and application target.

- The time dependent output voltages measured in Figs. 13 and 14 show a significant level of noise which consists of the intrinsic noise sources in a conventional circuits such as thermal noise, 1/f noise [32] and also the fluctuation of corona discharge. Indeed, since the discharge mechanism is different from the positive corona to negative one, the applied voltage must be appropriately selected to ensure the stability of a glow state positive corona or a pulseless negative corona [33]. In addition, a signal conditioning circuit, commonly used in the field of sensors, will also help reduce the noise level.

- As we know, the mobility of ion wind depends on the temperature and pressure and can be determined using the Mason-Schamp equation for the ion mobility as $[\mu \propto T / P]$, where $\mathrm{T}$ and $\mathrm{P}$ are the temperature and pressures, respectively. This relation depicts that, when the mobility of ion wind is decreasing at a lower temperature, a higher voltage is necessary to restore the initial discharge current. 


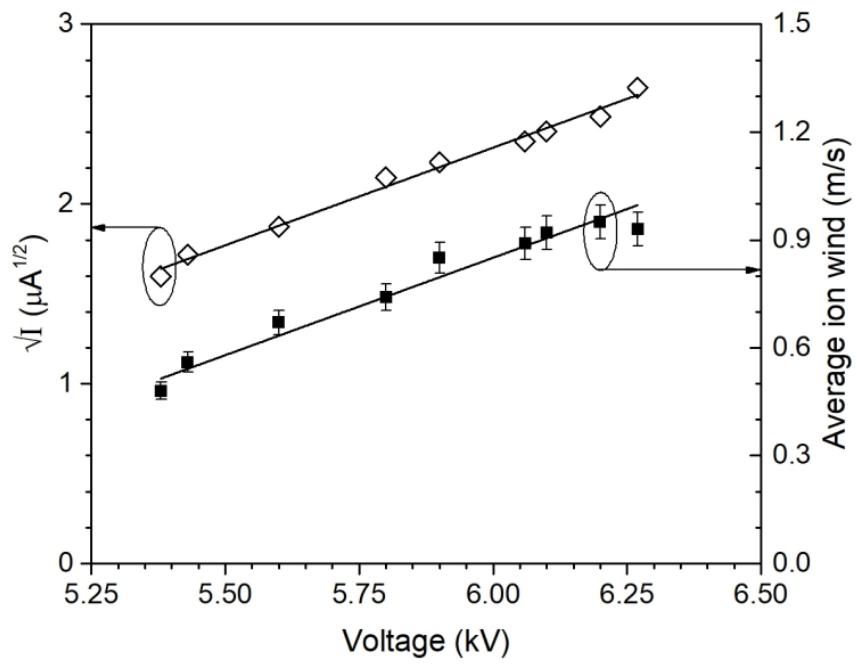

Figure 16. Characteristics of the negative corona using the configuration with grounded pin and positive ring electrodes and an inter-electrode distance of $7 \mathrm{~mm}$ : Relationships of $(U \propto \sqrt{I})$ and $(U \propto V)$.

- Since the present device is an open system, air from the outside would move into the sensing chamber and affect the voltage measured on the hotwires when the device rotates. However, ion wind at high speeds causes a positive pressure inside the sensing chamber, hence preventing the influx of air. In fact, results by both experimental and numerical works show that the impact of the environment on the device is insignificant if the system operates with a discharge voltage more than $5 \mathrm{kV}$ and a maximum angular speed of $10 \mathrm{rpm}$. Nevertheless, a closed system would be more preferable and will be considered in our future work.

- The sensing range of this sensor is experimental validated at $10 \mathrm{rpm}$ (i.e., $\pm 60 \% \mathrm{~s}$ ) which is suitable for several low motion applications in ship stabilization or motorcar as shown in [34] and is also comparable with fluidic angular rate sensor which was recently developed by [7]. In addition, the performance of the present system can be enhanced using our techniques which have been recently developed including the use of silicon doped hotwire in place of tungsten [6], [7], or further boosted with a p-n junction temperature sensing element [35].

- Finally yet importantly, the energy consumption of the whole system is low. The total power consumed by the whole system is around $75 \mathrm{~mW}$ including $15 \mathrm{~mW}$ for corona discharge process. It is relatively lower than that by the designed system using the conventional piezoelectric diaphragm, which requires a power of 200mW [36][38].

\section{Conclusions}

We have reported a new design of jet flow gyroscope using ion wind corona discharge by a numerical simulation and experimental analysis with the configuration of pin - ring electrodes. The deflection of ion wind jet flow by the Coriolis force from the angular rate is detected with a sensitivity of $4.7 \mu \mathrm{V} / \%$ s. The device is robust because the new structure does not require any vibrating component using instead the characteristic of ion wind jet flow. Furthermore, due to low energy consumption, a small battery can be used for the present ion wind gyroscopes. Finally, another benefit of this work is to contribute to valuable sensing techniques and the manipulation of fluidics for multidisciplinary fields such as microfluidics, analytical chemistry, and a "lab-on-a-chip".

\section{ACKNOWLEDGEMENT}

This research is funded by Vietnam National Foundation for Science and Technology Development (NAFOSTED) under grant number 107.01-2015.22.

\section{REFERENCE}

[1] A. P. French, Newtonia Mechanics. New York, NY, USA: Norton, 1971.

[2] R. Zhu, H. Ding, Y. Su, and Y. Yang, "Modeling and experimental study on characterization of micromachined thermal gas inertial sensors," Sensors, vol. 10, no. 9, pp. 8304-8315, 2010.

[3] J. Bahari, R. Feng, and A. M. Leung, "Robust MEMS Gyroscope Based on Thermal Principles," J. Microelectromechanical Syst., vol. 23, no. 1, pp. 100-116, Feb. 2014. 
[4] R. Mukherjee, J. Basu, P. Mandal, and P. K. Guha, "A review of micromachined thermal accelerometers," J. Micromechanics Microengineering, vol. 27, no. 12, p. 123002, Dec. 2017.

[5] V. T. Dau, T. X. Dinh, and S. Sugiyama, "A MEMS-based silicon micropump with intersecting channels and integrated hotwires," J. Micromechanics Microengineering, vol. 19, no. 12, p. 125016, Dec. 2009.

[6] V. T. Dau, D. V. Dao, T. Shiozawa, H. Kumagai, and S. Sugiyama, "A Single-Axis Thermal Convective Gas Gyroscope," Sensors Mater., vol. 17, no. 8, pp. 453-463, 2005.

[7] V. T. Dau, D. V. Dao, T. Shiozawa, H. Kumagai, and S. Sugiyama, "Development of a dual-axis thermal convective gas gyroscope," J. Micromechanics Microengineering, vol. 16, no. 7, pp. 1301-1306, Jul. 2006.

[8] V. T. Dau, T. X. Dinh, D. V. Dao, and S. Sugiyama, "Design and Simulation of a Novel 3-DOF MEMS Convective Gyroscope," IEEJ Trans. Sensors Micromachines, vol. 128, no. 5, pp. 219-224, May 2008.

[9] P. T. Hoa, T. X. Dinh, and V. T. Dau, "Design Study of Multidirectional Jet Flow for a Triple-Axis Fluidic Gyroscope," IEEE Sens. J., vol. 15, no. 7, pp. 4103-4113, Jul. 2015.

[10] K. Mori, H. Yamamoto, K. Takemura, S. Yokota, and K. Edamura, "Dominant factors inducing electro-conjugate fluid flow," Sensors Actuators A Phys., vol. 167, no. 1, pp. 84-90, 2011.

[11] K. Takemura, S. Yokota, M. Suzuki, K. Edamura, H. Kumagai, and T. Imamura, "A liquid rate gyroscope using electro-conjugate fluid," Sensors Actuators A Phys., vol. 149, no. 2, pp. 173-179, 2009.

[12] Y. Ogawa, S. Yokota, K. Edamura, and K. Takemura, "A Dual-Axis Liquid Micro Rate Gyroscope Using an Electro-Conjugate Fluid," Trans. JAPAN Soc. Mech. Eng. Ser. C, vol. 77, no. 773, pp. 204-211, 2011.

[13] V. T. Dau, T. X. Dinh, C. D. Tran, P. N. Bui, D. D. Vien, and H. T. Phan, "Fluidic mechanism for dual-axis gyroscope," Mech. Syst. Signal Process., vol. 108, pp. 73-87, 2018.

[14] H. Phan, T. Dinh, P. Bui, and V. Dau, "Transient Characteristics of a Fluidic Device for Circulatory Jet Flow," Sensors, vol. 18 , no. 3, p. 849, Mar. 2018.

[15] A. P. Chattock, "On the velocity and mass of the ions in the electric wind in air," Philos. Mag. Ser. 5, vol. 48, no. 294, pp. 401420, Nov. 1899.

[16] E. Moreau, "Airflow control by non-thermal plasma actuators," J. Phys. D. Appl. Phys., vol. 40, no. 3, pp. 605-636, Feb. 2007.

[17] D. B. Go, T. S. Fisher, and S. V Garimella, "Direct simulation of ionization and ion transport for planar microscale ion generation devices," J. Phys. D. Appl. Phys., vol. 42, no. 5, p. 55203, 2009.

[18] T. C. Corke, C. L. Enloe, and S. P. Wilkinson, "Dielectric Barrier Discharge Plasma Actuators for Flow Control*," Annu. Rev. Fluid Mech., vol. 42, no. 1, pp. 505-529, Jan. 2010.

[19] A. Belinger, N. Naudé, J. P. Cambronne, and D. Caruana, "Plasma synthetic jet actuator: electrical and optical analysis of the discharge," J. Phys. D. Appl. Phys., vol. 47, no. 34, p. 345202, 2014.

[20] Y. Fukatsu, E. Nomura, and K. Matsu, "Gas rate gyro," US4941353, 1990.

[21] Y. Cai and Y. Zhao, "Ion discharge gyroscope," US8146423, 2012.

[22] M. Robinson, "Movement of air in the electric wind of the corona discharge," Trans. Am. Inst. Electr. Eng. Part I Commun. Electron., vol. 80, no. 2, pp. 143-150, 1961.

[23] G. F. L. Ferreira, O. N. Oliveira, and J. a. Giacometti, "Point-to-plane corona: Current-voltage characteristics for positive and negative polarity with evidence of an electronic component," J. Appl. Phys., vol. 59, no. 9, pp. 3045-3049, 1986.

[24] W. L. Lama and C. F. Gallo, "Systematic study of the electrical characteristics of the 'trichel' current pulses from negative needle-to-plane coronas," J. Appl. Phys., vol. 45, no. 1, pp. 103-113, 1974.

[25] L. Li, S. J. Lee, W. Kim, and D. Kim, "An empirical model for ionic wind generation by a needle-to-cylinder dc corona discharge," J. Electrostat., vol. 73, pp. 125-130, 2015.

[26] I. Mabuchi and T. Tanaka, "Experimental Study on Effect of Vibration on Natural Convective Heat Transfer from a Horizontal Fine Wire," Bull. JSME, vol. 10, no. 41, pp. 808-816, Feb. 1967.

[27] V. T. Dau, T. X. Dinh, T. T. Bui, C. D. Tran, H. T. Phan, and T. Terebessy, "Corona based air-flow using parallel discharge electrodes," Exp. Therm. Fluid Sci., vol. 79, pp. 52-56, 2016.

[28] V. T. Dau, C. D. Tran, T. T. Bui, V. D. X. Nguyen, and T. X. Dinh, "Piezo-resistive and thermo-resistance effects of highly-aligned CNT based macrostructures," RSC Adv., vol. 6, no. 108, pp. 106090-106095, 2016.

[29] T. X. Dinh, D. B. Lam, C.-D. Tran, T. T. Bui, P. H. Pham, and V. T. Dau, "Jet flow in a circulatory miniaturized system using ion wind," Mechatronics, vol. 47, no. September, pp. 126-133, Nov. 2017.

[30] V. T. Dau, T. X. Dinh, T. T. Bui, and T. Terebessy, "Corona anemometry using dual pin probe," Sensors Actuators A Phys., vol. 257, pp. 185-193, 2017.

[31] A. Yehia and A. Mizuno, "Ozone generation by negative direct current corona discharges in dry air fed coaxial wire-cylinder reactors," J. Appl. Phys., vol. 113, no. 18, p. 183301, 2013.

[32] F. N. Hooge, "1/f noise is no surface effect," Phys. Lett. A, vol. 29, no. 3, pp. 139-140, Apr. 1969.

[33] J. S. Chang, P. a. Lawless, and T. Yamamoto, "Corona discharge processes," IEEE Trans. Plasma Sci., vol. 19, no. 6, pp. 11521166, 1991.

[34] "Attitude Measurement Unit for motorcars | Products - Gyros | TAMAGAWA SEIKI CO.,LTD." [Online]. Available: https://www.tamagawa-seiki.com/products/gyro/automotive-attitude-measurement-sensor.html. [Accessed: 10-Apr-2018].

[35] V. T. Dau, D. V. Dao, T. Shiozawa, and S. Sugiyama, "Convective Gas Gyroscope Based on Thermo-Resistive Effect in Si P-N Junction," in TRANSDUCERS 2007 - 2007 International Solid-State Sensors, Actuators and Microsystems Conference, 2007, pp. $2525-2528$.

[36] V. T. Dau, T. X. Dinh, and T. T. Bui, "Jet flow generation in a circulatory miniaturized system," Sensors Actuators B Chem., vol. 
223, pp. 820-826, 2015.

[37] H. T. Phan, T. X. Dinh, and V. T. Dau, "Development of a jet-generator and its application to angular rate sensor," in 2015 China Semiconductor Technology International Conference, 2015, pp. 1-3.

[38] L. Murata Manufacturing, "Piezoelectric Diaphragms, Murata Manufacturing Co., Ltd.," 2016. [Online]. Available: http://www.murata.com/en-sg/products/sound/diaphragm. 
Van Thanh Dau received the B.S. degree in aerospace engineering from Hochiminh City University of Technology, Vietnam, in 2002, and the M.Sc. and Ph.D. degrees in micro-mechatronics from Ritsumeikan University, Japan, in 2004 and 2007, respectively. He has been with the Research Group, Sumitomo Chemical, Japan since 2010, exploring the integrated micro fluidic atomization methods. He has authored over 100 papers in scientific journals and conference proceeding. He has filed over 30 intellectual properties, been granted seven US and many international patents. His research interests include microfluidics, sensors and actuators, particle generation and electro-fluido-dynamic.

Dr. Dau was a recipient of the Japan Society for the Promotion of Science Post-Doctoral Fellowship from 2007 to 2009 . He was a visiting scholar at the National Institute of Advanced Industrial Science and Technology, Japan in 2009, and a visiting researcher at Atrium Innovation, Ltd. Oxford-shire, United Kingdom in 2011.

Thien Xuan Dinh received the B.S. degree in aerospace engineering from Hochiminh City University of Technology in 2002, Vietnam and the M.Sc. and Ph.D. degrees in mechanical engineering from Ritsumeikan University in 2004 and 2007 , respectively. He was recipient of Japan Government Scholarship (MEXT) for Outstanding Student to pursuits his M. Sc. and $\mathrm{Ph}$. D. courses and Japan Society for the Promotion of Science postdoctoral fellowship from 2011 to 2013.

His general research interest is computation of fluid flow. The large parts of his research are turbulence modeling using Large Eddy Simulation, multiphase modeling using Volume of Fluid technique, and simulation of turbulence and dispersion. Recently, he has focused on computation of fluid flow for developing microfluidic devices as electrohydrodynamics, microsensors, micropump, and micromixer for biochemical engineering.

Canh-Dung Tran (CD Tran) is currently working as a Senior Lecturer at School of Mechanical and Electrical Engineering, Faculty of Health, Engineering and Sciences, Senior Research Fellow of the Computational Engineering Scientific Research Centre, The University of Southern Queensland (USQ), Australia; and Research Fellow at the Department of Mechanical Engineering, National University of Singapore.

From 2005 to 2010, he was a Research Scientist and Project Leader at the CSIRO and participated in various projects relating to modelling and analyzing the mechanical behavior of materials. He has gained wide experience in developing mechanical, material engineering and Carbon Nano-tube research proposals, which were funded internally and externally, in conjunction with the CSIRO, University of Texas at Dallas (USA), University of Sydney (Australia) and the University College of London (UK). Supported by a USQ scholarship, he earned his PhD degree in Mechanical Engineering in 2004. In 1994, he received a national scholarship of the French Government to complete his research DEA program on Mechanics of Advanced Fibrous Materials at the University of Haut Alsace, France. He has produced about 100 research publications as author and co-authors in the areas of numerical simulation including stochastic macro-micro methods, advanced Fibrous Materials, nanomaterials and CNTs.

Bui Thanh Tung received the B.S. degree in electrical engineering from Vietnam National University, Hanoi (VNUH) in 2004, and the M.E. and D.Eng. degrees in Science and Engineering from Ritsumeikan University, Shiga, Japan, in 2008 and 2011, respectively. From 2011 to 2015 he was a post-doctoral researcher with the 3D Integration System Group, Nanoelectronics Research Institute (NeRI), National Institute of Advanced Industrial Science and Technology (AIST), Tsukuba, Japan. Currently, he is an assistant professor at the Faculty of Electronics and Telecommunication (FET), University of Engineering and Technology (UET), Vietnam National University, Hanoi (VNUH). His current interests include MEMS based sensors, actuators and applications.

Phan Thanh Hoa received the B.E degree in electronic engineering from Hanoi University of Science and Technology, Vietnam, from 1999. He acquired the M.Sc. and Ph.D. in electronic engineering from Ritsumeikan University, Japan, in 2003 and 2012, respectively. Since 2012, he joined the Hanoi University of Industry (HaUI), where he became a lecturer of Department of Electronic and Telecommunication, Faculty of Electronic engineering, and Head of Department of Electronic and Energy Engineering at HaUI Institute of Technology. His research interests are in MEMS-based sensor devices, intelligent transportation systems, wireless sensor network, and resource allocations and QoS of mobile broadband network. 


\section{Manuscript Details}

\section{Manuscript number}

Title

Article type
SNA_2018_53_R2

A study of angular rate sensing by corona discharge ion wind

Research Paper

\section{Abstract}

We report an application of ion wind in angular rate sensing. A new design of a jet flow gyroscope using ion wind corona discharge is developed by conducting a numerical simulation and then an experimental analysis with regard to the advantages of a corona-discharge-based jet flow. Ion wind is generated by applying a high-voltage between a pin, as the discharge electrode, and a ring, as the reference electrode. The current-voltage characteristics of the discharge process by experimental works is set up as the boundary condition to describe the ion wind flow in the numerical simulation. A jet flow of ion wind is observed through an experiment and a simulation. When the gyroscope is subjected to an angular rate, the induced Coriolis force deflects the ion wind. This deflection is detected using four hotwires installed downstream of the working chamber behind the reference electrode. The experimental result show that the angular rate can be detected with a sensitivity of $4.7 \mu \mathrm{V} / \mathrm{o} / \mathrm{s}$. Because ion wind can be generated with minimum power and does not require any vibrating components, the device is robust, consumes low power, and is cost-effective.

\section{Keywords}

Manuscript category

Corresponding Author

Corresponding Author's Institution

Order of Authors

Suggested reviewers ion wind; angular rate sensing; OpenFOAM; dielectric barrier

Physical Sensors

Van Dau

Health \& Crop Sciences Research Laboratory

Van Dau, Thien Dinh, Canh-Dung Tran, Tung Thanh Bui, Hoa Phan Thanh

Shiqiang Liu, Beelee Chua, Rong Zhu, chong shen

\section{Submission Files Included in this PDF}

\section{File Name [File Type]}

D526b_CoverLetter_revised.doc [Cover Letter]

D526b_Response to reviewer' comments_revised.docx [Response to Reviewers]

d526b_Highlight_revised.doc [Highlights]

d526b_1Abstract_revised.docx [Abstract]

d526b_1CoronaGyro_Manuscript_revised.docx [Manuscript File]

Photograph and biographies_revised.docx [Author Biography]

To view all the submission files, including those not included in the PDF, click on the manuscript title on your EVISE Homepage, then click 'Download zip file'. 